\title{
Drying kinetics of cellulose nanofibers suspensions
}

\section{Al Zaitone, B.}

Department of Chemical and Materials Engineering, King Abdulaziz University, Jeddah, Kingdom of Saudi Arabia

*E-mail of the corresponding author: balzaitone@kau.edu.sa

\begin{abstract}
Cellulose nanofibers (CNF) is used in various pharmaceutical applications due to its unique characteristics i.e., biodegradability, mechanical and biological properties. CNF is often produced by spray drying process, knowledge of the drying kinetics in terms of mass and heat transfer on the scale of single droplet is important for process development and model validation.

Acoustic levitator was used to study drying process of CNF suspension at different air temperatures and initial CNF concentrations. The unique property of acoustic levitation to hold single droplet contactless in the air, enables to study particle morphology during drying process, calculate evaporation rate and estimate particle porosity. Results show that packed particles result at lower initial concentration and temperature has a moderate influence on mean porosity of CNF dried particles.
\end{abstract}

Keywords: acoustic levitation; droplet; drying kinetics; Cellulose Nanofibers. 


\section{Introduction}

Cellulose nanofibers (CNF), is a promising biopolymer. The high surface area of CNF makes it suitable as a nanofiller to enhance the mechanical properties of composite materials [1]. The rheological and physio-chemical properties, allow CNFs to stabilize emulsions of different types and improve the drug delivery of active materials in poor soluble aqueous solutions [2]. The strength with low weight and high surface area make CNF a good candidate for capsule reinforcement [3], on the other hand CNF can play a significant role in producing sustained drug release formulation, the CNFs form a fiber network that hinder the diffusion of drug [3].

Cellulose nanofibers are mainly produced form cellulosic botanical sources such as wood, cotton, hemp or flax. The isolation of Cellulose nanofiber is achieved via mechanical or chemical processes, which result in gel-like liquid suspension that held cellulose nanostructures. CNFs are extracted from the aqueous medium via evaporation of the solution through lyophilization [4], supercritical drying and spray drying [5].

Spray drying is widely used in many industries, e.g. chemical, food and pharmaceutical processing. In spray drying, droplets generation is followed by solvent/liquid evaporation leading to a final powder product. The short retention time in spray drying, makes it ideal for heat sensitive materials. Controlling the drying kinetics is decisive to minimize the liquid amount in the particles and enhance good mechanical properties. Physico-chemical properties of the solution together with operating parameters, greatly influence drying kinetics.

Since experiments on real sprays are difficult to interpret and only integral information about an ensemble of droplets can be obtained, simple and idealized configurations considering single droplets are often preferred for investigation of such spray drying situations.

The experimental work presented in this paper deals with the drying process of individual CNF suspension droplets. The focus of drying experiment was to determine the drying kinetics at various process parameters i.e., gas drying temperature, CNF loading. The acoustic levitation has been used, exhibiting an almost steady positioning of the sample; hence providing a unique opportunity to observe a droplet throughout the entire drying period. The imaging technique allows for accurate measurement of temporal change of droplet diameter and vertical size, that were used to calculate drying rate and mass of the droplet during the drying process and estimation of dried particle porosity.

The paper is organized as follows: in the next section, materials and methods i.e. acoustic levitation are described and the influence of the acoustic streaming on liquid evaporation is discussed, thereafter, the experimental and theoretical results are presented. The paper ends with the conclusions. 


\section{Materials and Methods}

\subsection{Materials}

The CNF used for the microparticles production was obtained from Process Development Center University of Maine (Orono, USA) in form of 3.0\% aqueous gel. The nominal fiber diameter is $50 \mathrm{~nm}$ and fiber length up to few hundred micrometers. Deionized distilled water was used to prepare CNF suspension.

\subsection{Methods}

\subsubsection{Preparation of CNF suspension for single droplet drying}

The CNF aqueous gel solid content was proofed by drying 10 different samples of CNF aqueous gel in an oven at $60{ }^{\circ} \mathrm{C}$ for 10 hours, the mass fraction of dry fibers was estimated to be $3.5 \% \pm 0.13$. The desired suspensions were prepared by diluting CNF aqueous gel using deionized distilled water. CNF suspension was homogenized using magnetic stirrer for 30 mins to assure homogeneity of the cellulose fibers suspensions. The desired concentrations of CNF suspension were 2,4 and $6 \mathrm{mg}(\mathrm{CNF}) / \mathrm{ml}$ (water).

\subsubsection{Single droplet drying apparatus}

The ultrasonic levitator was used for the drying experiments; the main setup is sketched in Fig. 1, it consists of an acoustic levitator which operates at $58 \mathrm{kHz}$ sound frequency. The emitted ultrasonic waves leave the transducer and propagate througth the gas mesdium to hit the concave reflector and then bounce to the transducer forming the so-called standing wave. The acoustic force generated by this standing wave is capable of stable positioning of the liquid or solid samples. The levitatator is equiped with heating chamber, and drying temperature up to $120^{\circ} \mathrm{C}$ of the gas can be achieved. The imaging system consists of a CMOS camera connected to a macro lens, it allows for monitoring the droplets. The droplet is illuminated via LED back light, the shadow image captured by the camera is anaylzed online to calculate droplet equivalent diameter, vertical position and aspect ratio. A microliter syringe with long needle is used to insert axially the droplet into the acoustic field.

\section{Influence of the acoustic field}

The interaction between the acoustic field and CNF droplets suspended by the acoustic force generates streaming flow around the droplet. Several authors $[6,7]$ studied the induced flow and quantify its effect on the evaporation of the liquid. The acoustic streaming were first defined Schlichting [8], these streaming are divided into two categories; the inner acoustic streaming which drives the mass and heat transfer between the droplet surface and the surrounding gas medium $[6,9]$. 


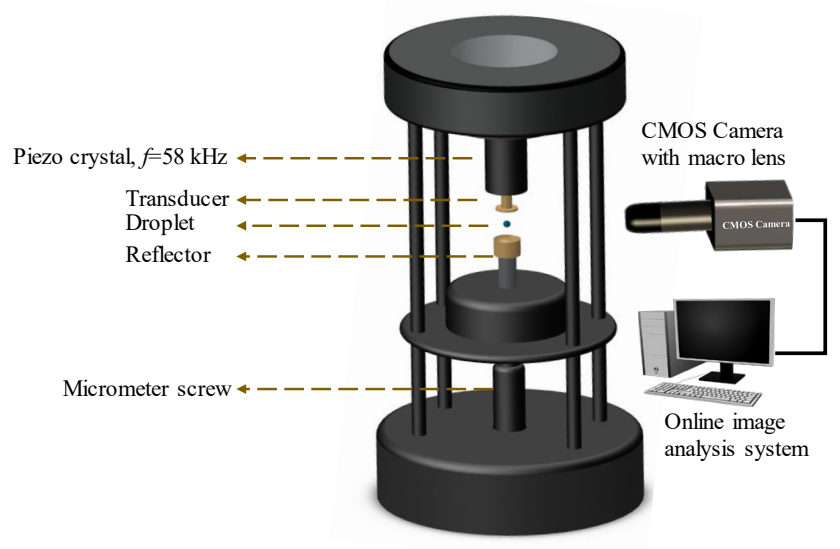

Fig. 1 Schematic picture of the acoustic levitator.

The second streaming flow is called outer acoustic streaming which represent toroidal vortices, these vortices trap the evaporated liquid from the droplet. Therefore, the vapor is accumulated around the droplet and alter the far-field vapor concentration. As more vapor is trapped by the outer acoustic streaming, the evaporation rate is reduced and comparison with model become rather difficult. A depletion of the accumulated vapor from such vortices becomes mandatory to have reproducible results and enable model validation. An air flow of $0.85 \mathrm{l} / \mathrm{min}$ is inserted surrounding the droplet to destroy the outer acoustic streaming and remove the vapor accumulated around the droplet [10].

\section{Results and Discussion}

\subsection{NFC Microparticle production}

CNF has strong affinity to water, at very small CNF concentration ca. $3.5 \%$ wt., a slurry is formed, in order to generate small droplets using the microliter syringe, suspension at very low CNF loading were prepared, namely 2,4, and $6 \mathrm{mg} \mathrm{CNF}$ per $\mathrm{ml}$ of water.

The drying temperature was varied in the range $\left(20-80{ }^{\circ} \mathrm{C}\right)$, droplets of $1.0-2.0 \mu \mathrm{l}$ of the suspension was introduced into the acoustic field, once the drying experiment finished the particle was collected to study its morphology and surface structure under scanning electron microscopy. As shown in Fig. 2, the drying of CNF suspensions droplet results in water evaporation and an agglomerate of fibrous structure is formed. The initial droplet size was $1.5 \mathrm{~mm}$. at the end of drying course in the acoustic levitator, the dried particle size is reduced to ca. 200-300 $\mu \mathrm{m}$ diameter and an agglomerate of the nanofibers was obtained. It can be seen that particles are not spherical, Fig. 2a shows the surface morphology of the particle where the nanofiber consolidated together, Fig. $2 \mathrm{c}$ depicts the internal structure of the particle after it has been broken, the porous structure and the random alignment of the fibers can be 
easily noticed, despite the length of the cellulose nanofibers, they have been folded inside the droplet due to high surface tension of the water comparing to the soft cellulose nanofibers structure.

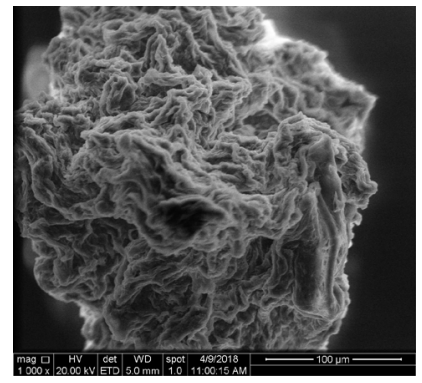

a)

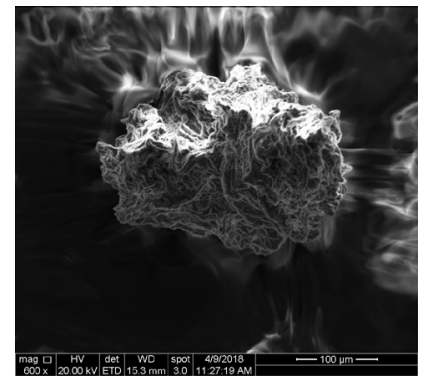

b)

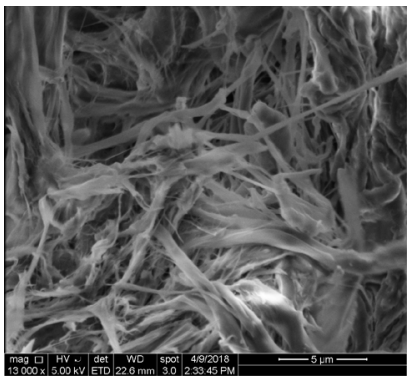

c)

Fig. 2 Scanning Electron Microscopy images of dried cellulose nanofibers suspensions a) $6 \mathrm{mg} / \mathrm{ml} \mathrm{at}$ $\left.80^{\circ} \mathrm{C} \mathrm{b}\right) 6 \mathrm{mg} / \mathrm{ml} \mathrm{at} 60^{\circ} \mathrm{C}$ and c) internal structure of broken particle.

\subsection{Evaporation rate and porosity estimation}

The drying rate and the mean porosity of dried particle is disused in this section. Four different temperatures were investigated, the surface decay of the droplets is shown in Fig. 3, the higher the temperature is, the droplet drying rate becomes faster. The experiments in Fig. 3 were conducted with the same initial volume namely $2 \mu l$ and different loading of CNF was used. The drying curves in terms of normalized surface area decay can be divided into two stages, the first one, where the loading of CNF is very low, mass fraction of CNF is less than $0.05 \%$ wt., the evaporation rate here follows the well-known $\mathrm{d}^{2}$-law. Actually, for small CNF loading, the drying curve is similar to the evaporation of pure water, once the loading of CNF increases, it starts competing with the available sites for water evaporation, as more of the solids accumulated on the surface, the evaporation of the liquid becomes slower i.e., second stage begins. Finally, the droplet forms an agglomerate of cellulose nanofibers, the size of the particle remains constant.

The evaporation rate was calculated from the slope of the temporal evolution of the surface decay curves and represented by a solid line in Fig. 3.

The porosity of the dried particle is an important parameter for modelling drying kinetics, the water passes through particle voids to reach the surface. On the other hand CNF can play a significant role in producing sustained drug release formulation, the CNFs form a fiber network that hinder the diffusion of drug [3]. At lower particle porosity, the drug diffusion become slower, porosity is calculated using the equation:

$$
\varepsilon=1-\frac{m_{s}}{\rho_{s} V_{p}}
$$


Where, $\varepsilon$ is the porosity, $\mathrm{m}_{\mathrm{s}}, \rho_{\mathrm{s}}$ is the mass and density of solids respectively, $\mathrm{V}_{\mathrm{p}}$ is the volume of the particle in $\mathrm{m}^{3}$. As depicted in Fig. 3, higher porosity is obtained for lower initial CNF concentration, while temperature has a moderate influence on mean particle porosity.

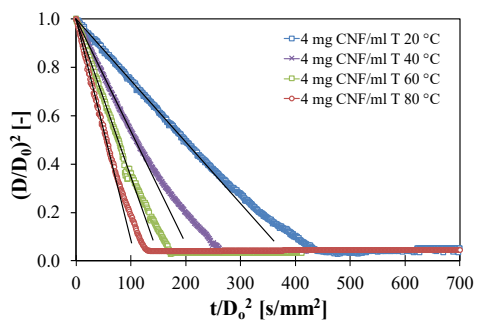

a)

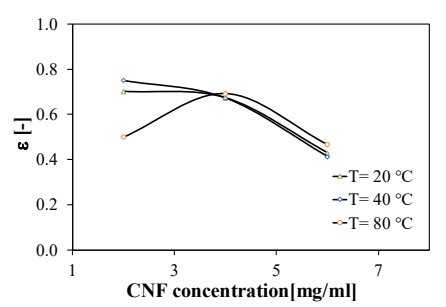

c)

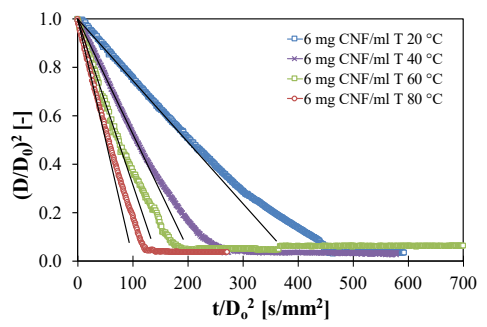

b)

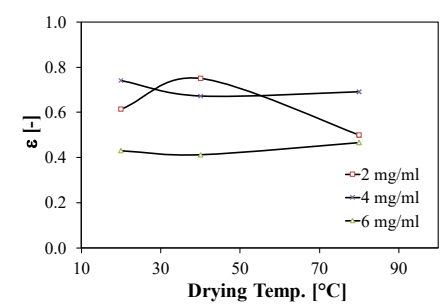

d)

Fig. 3 Temporal evolution of surface area decay of CNF suspension droplet for a) $4 \mathrm{mg} C \mathrm{CNF} / \mathrm{ml}$, b) 6mg $\mathrm{CNF} / \mathrm{ml}$ at $20,40,60$ and $80{ }^{\circ} \mathrm{C}$, the initial droplet volume is $2.0 \mu \mathrm{ll}$ c) \& d) calculated mean porosity of dried CNF particle as a function of CNF loading and drying temperature, respectively.

\subsection{Constant and falling rate periods}

Suspension of CNF droplet exhibit upon course of drying changes in physical properties, i.e., density of the droplet increases as more liquid is evaporated, the shape of the droplet changes until the final morphology evolves at the end of the drying. In case of mass transfer rate, droplet drying shows in general two regimes, the first one is called constant rate period and the second one is falling rate period $[11,12]$.

In constant rate period the evaporation of the liquid occurs from a wet particle surface. As depicted in Fig. 4, evaporation of the liquid is accompanied by size reduction i.e., droplet normalized surface area decreases with time. The shrinkage of droplet continues, and the concentration of CNF increases until a small particle or grain of CNF is formed. During the constant rate period the evaporation rate occurred occurs at constant wet bulb temperature, the evaporation rate is calculated form the volume change of the droplet of the liquid:

$$
\left.\frac{d m_{d, c}}{d t}\right|_{\text {const }}=-\rho_{d} \frac{\Delta V}{\Delta t}
$$

Where $\mathrm{V}$ is the volume of the droplet in $\mathrm{m}^{3}$ and $\rho_{d}$ is droplet density in $\mathrm{kg} / \mathrm{m}^{3}$. 
As the liquid evaporates, the droplet alters its vertical position in the acoustic field. The vertical position is function of droplet's volume and weight [6], it rises in the acoustic field towards pressure node as shown in Fig. 4.

Once the volume and aspect ratio of the droplet/particle seized, the constant rate period comes to its end and the falling rate period starts, this is marked by constant surface area in Fig. 4, here, the shadow image technique is not able any more to deliver information about the mass transfer rate because the particle/grain has now a constant volume that is calculated from its meridional section.

The evaporation of the liquid continues and by monitoring the vertical position of the grain in the acoustic field, further information about the mass transfer can be found. The acoustic force acts against gravity force and once the droplet volume is constant, any decrease in particle weight due to liquid evaporation force the droplet to move upwards to the adjacent pressure node.

The mass of the particle can be easily found by interpolation of the vertical position in the falling rate period, knowing that the rise of the particle in the acoustic field is now only function of the density.

$$
m_{d, f}(t)=\frac{\Delta m_{f, \max }}{\Delta y_{f, \max }} \cdot\left(\text { vert.pos }(t)-\text { vert.pos }\left(t_{f 0}\right)\right)+m_{d, c}
$$

Where, $\mathrm{m}_{\mathrm{d}, \mathrm{f}}$ is the mass of droplet in the falling in $\mathrm{kg}, \Delta m_{f, \max }$ and $\Delta y_{f, \max }$ are maximum difference of particle mass and particle vertical distance respectively and calculated between the beginning and the end of the falling rate period. $m_{d, c}$ is the mass of droplet at the end of constant rate period. The mass of the liquid at the end of falling rate period is approximately zero, $\mathrm{t}_{\mathrm{f} 0}$ is the time in seconds when falling rate period begins.

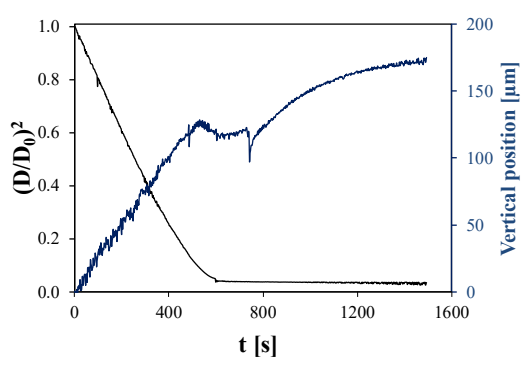

a)

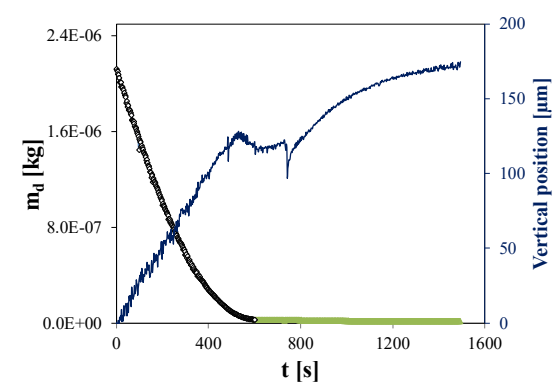

b)

Fig. 4 Vertical position of the droplet (CNF $\left.6 \mathrm{mg} / \mathrm{ml} \mathrm{TE} 40^{\circ} \mathrm{C}\right)$ during the course of drying, the first stage of drying is designated by volume reduction of the droplet and the second stage of drying is designated by rise in the acoustic field at constant volume. 
The above results show the importance of the acoustic levitator to measure the drying kinetics of CNF suspension droplets, and its ability to measure particle's drying rate in both constant and falling rate periods. The estimated porosity of dried particle which is an important product property and was obtained for different drying temperatures and CNF loadings.

\section{Conclusions}

The drying kinetics and porosity of CNF dried particle has been investigated, a series of experiments were conducted in the acoustic levitator to mimic the spray drying process occurred in spray dryer. Drying rates and the evolution of particle morphology were monitored using the shadow imaging technique. Particle shape and inner structure of cellulose nanofibers were analysed by SEM technique. The drying rate of droplet increases at high temperatures. The porosity of the dried particles shows an increase for higher CNF initial concentrations and drying temperature has a moderate influence on particle porosity.

\section{References}

[1] Santos, F., Iulianelli, G. C. V., Inês, M., and Tavares, B. The Use of Cellulose Nanofillers in Obtaining Polymer Nanocomposites: Properties, Processing, and Applications. Materials Sciences and Applications 2016, 7 (5), 257-294.

[2] Löbmann, K., and Svagan, A. J. Cellulose nanofibers as excipient for the delivery of poorly soluble drugs. International Journal of Pharmaceutics 2017, 533 (1), 285-297.

[3] Kolakovic, R., Laaksonen, T., Peltonen, L., Laukkanen, A., and Hirvonen, J. Spraydried nanofibrillar cellulose microparticles for sustained drug release. International Journal of Pharmaceutics 2012, 430 (1-2), 47-55.

[4] Han, J., Zhou, C., Wu, Y., Liu, F., and Wu, Q. Self-assembling behavior of cellulose nanoparticles during freeze-drying: Effect of suspension concentration, particle size, crystal structure, and surface charge. Biomacromolecules 2013, 14 (5), 1529-1540.

[5] Peng Y, Y. H. G. J. D. Spray-drying cellulose nanofibrils: effect of drying process parameters on particle morphology and size distribution. Wood and Fiber Science 2012, 44 (4), 1-14.

[6] Yarin, A. 1., Brenn, G., Kastner, O., Rensink, D., and Tropea, C. Evaporation of acoustically levitated droplets. Fluid Mech. 1999, 399, 151-204.

[7] Trinh, E. H., and Robey, J. L. Experimental study of streaming flows associated with ultrasonic levitators. Phys. Fluids 1994, 6 (11), 3567-3579.

[8] Schlichting, H. Boundary Layer Theory, McGraw-Hill, Inc., 1978.

[9] Gopinath, A., and Mills, A. F. Convective Heat Transfer From a Sphere Due to Acoustic Streaming. Journal of Heat Transfer 1993, 115 (2), 332-341.

[10] Al Zaitone, B. A., and Tropea, C. Evaporation of pure liquid droplets: Comparison of droplet evaporation in an acoustic field versus glass-filament. Chemical Engineering Science 2011, 66 (17), 3914-3921.

[11] Nesic, S., and Vodnik, J. Kinetics of droplet evaporation. Chemical Engineering Science 1991, 46 (2), 527-537.

[12] Mujumdar, A. S. Handbook of Industrial Drying, Marcel Dekker, New York, 2006. 